\title{
Mining Potential Requirements by Calculation of User Operations
}

\author{
Yanqun Huang, Tianjin University, China* \\ Gaofeng Pan, Tianjin Tianduan Press Co. Ltd., China \\ $\mathrm{Xu} \mathrm{Li}$, Tianjin Polytechnic University, China \\ Zhe Sun, Tianjin University, China \\ Shinichi Koyama, University of Tsukuba, Japan \\ Yanqun Yang, Fuzhou University, China
}

\begin{abstract}
This study proposes a method for mining potential user requirements from users' nonverbal behaviors by analyzing their operational problems, since human behaviors reflect emotions and operational bottlenecks in human-machine interactions. Taking a single daily operation task as an example, three key steps were included in the method: first, modeling users' operation and constructing the operation chain; second, finding emotional or physical problems in the operation chain, where the problems were defined mathematically as an emotional or physical load at each suboperation; and third, defining and obtaining potential user requirements by improving the operational problems when performing a task. Furthermore, a daily operation task was introduced to demonstrate and validate the method of mining user potential requirements. The results indicate that it is effective to discover the potential needs for a specific product and provide satisfactory solutions by calculating and optimizing operational problems.
\end{abstract}

\section{KEYWORDS}

Human-Machine Interaction, Nonverbal Behavior, Operations, Potential Requirements, Requirement Mining, Single Task, User, User Experience

\section{INTRODUCTION}

People regularly experience negative emotions such as embarrassment, failure, difficulty, and risk, when they fail to do something, for example, bumping into something, or not keeping their house organized, because they think it is their fault for not completing a task perfectly. Most people cannot voice their need for specific new tools, products, or operation patterns required to accomplish a task more comfortably (Leonard, 2013). For example, pregnant women and the elderly may have difficulty putting on shoes; children may be allergic (Turan et al., 2011) or psychologically sensitive to toilet seats, and might even experience genitourinary injury owing to an accidental fall off a toilet seat (Glass et al., 2013); and drivers might spend a few minutes trying to maintain a constant comfortable car temperature when starting a car. People take these simple things for granted and even blame themselves for carelessness or clumsiness instead of asking for a new product (Norman, 2013). Thus, it is difficult to obtain users' implicit potential requirements directly from their verbal expressions. 
Users' behaviors reflect emotions and behavioral bottlenecks in operations on a product, which provide key clues for conception generation. Many studies have made progress in inferring potential requirements indirectly from user behaviors, for example, by affection analysis (Shin et al., 2020), netnography (Liao et al., 2021), and online product choice/reviews (Lai et al., 2019). Most of these studies are focused on indirect extrapolation of general trends from cases, while few have focused on the modeling of user requirements by analyzing users' nonverbal behavior to help particular potential requirements mining in particular operational environments, because of the uncertainty and complexity of the operation process. The demanding problems in this domain include identifying the operations, expressing operational problems mathematically, defining users' potential requirements, and mining requirements from their nonverbal behaviors to generate creative ideas on convenient and user-friendly products.

\section{LITERATURE REVIEW}

User requirements can be categorized as explicit or implicit (Figure 1). For the former, users know what they need and can express it explicitly. Thus, user requirements can be obtained through verbal descriptions, such as questionnaires (Zelesniack et al., 2021; Zheng et al., 2018), interviews (Martin et al., 2012; Shukoor et al., 2018; Willard et al., 2018), and online feedback (Zanker \& Jessenitschnig, 2009). After collecting user requirements, their intentions can be translated into functional requirements. These methods include user experience-based research, such as trial run and usability assessment (Castellano-Tejedor et al., 2020; van Haasteren et al., 2020); and the evaluation of the degree of usability, mainly based on physiological ergonomic conflicts. Subsequently, user requirements are interpreted as new design solutions (Hansen \& Rosen, 2019; Sen \& Sener, 2020). These methods are effective in identifying explicit user requirements through quantitative or qualitative analyses.

Meanwhile, implicit requirements reflect users' potential intentions (Guo et al., 2019). According to the construct of subconsciousness in psychology (Leonard, 2013), most people do not know what they really need and like. Subsequently, instead of blaming the product, some people blame themselves when they fail in their daily jobs (Norman, 2013). For example, they might experience negative emotions if they miss the trash can when throwing trash away, dial the wrong phone number, drop a pen on the floor, or spill water on a keyboard; however, they do not expect different product designs to solve these problems.

Researchers have proposed many methods to understand implicit user requirements. Semantic differential (Osgood \& Luria, 1954; Petrenko \& Mitina, 2020) has been popularized in discovering users' emotional requirements (Klettner, 2020; Pauligk et al., 2019). Metaphorical extraction (Heath et al., 2014) was used to extract user expectations, including experiences, emotions, motivations, and values, by analyzing users' imaginations and explanations of certain images or scenes. Contextaware recommender systems have been effectively used to infer user preferences from user-generated textual reviews (Chen \& Chen, 2015). The lead user method (Sanchez et al., 2018) and typological scheme (Kakar, 2016) are other effective and widely used methods for capturing user requirements and predicting future product trends through digital anthropology or experimentation. In addition, KJ mining (Cheng \& Leu, 2011) has been used for product defect classification to ensure user satisfaction. With the rapid development of big data computing technology and data mining concepts in fog/cloud environments (Manasrah et al., 2019), intelligent algorithms are used to predict user requirements (Chen et al., 2019; Wang \& Zhang, 2017), such as in shopping systems (Kieu Que et al., 2019) and recommender systems (Silva-Rodriguez et al., 2020). These approaches can be generally divided into two categories: obtaining the relationship between user preferences and images or scenes according to past experiences, for example, semantic differential and metaphorical extraction; and extracting user preferences according to context-related information, for example, context-aware recommender system, KJ mining, lead user method and big data computing. These methods are based on an indirect 
extrapolation. They are helpful in predicting general product trends but imprecise for mining a specific potential requirement of a specific product or operation pattern.

Sun et al. (2018) created a function-task-behavior user manual and modeled user operations, where users were invited to participate in the co-creation process at the early stage of product concept formation. Additionally, the interaction between user behavior and product behavior was enabled. This approach emphasized the functional relationship between users and products; for example, a turning operation requires a turning structure. This led to a design conforming to user behavior, which started an approach of modeling user operations, and suggested a new innovative design method based on user operation behavior analysis. However, the potential requirements reflected by operational behaviors and problems have rarely been examined directly. Therefore, a new approach is needed to mathematically model implicit potential requirements according to users' operational behaviors and to improve user experiences.

In summary, the challenge is to quantitatively obtain users' implicit requirements from their behaviors, which involves the formulization of users' emotional status and physical obstacles when operating products, and then proposing strategies for improving users' emotional and physical experiences, including reducing emotional and physical loads according to human-centered design theory (Norman, 2013). This study aimed to examine users' potential requirements during a task performance procedure and ultimately contribute to providing a method for discovering clues of users' requirements for specific new operation patterns or new products. Finally, the modeling and optimization process was illustrated on a daily operation task.

Figure 1. Studies on requirements discovering

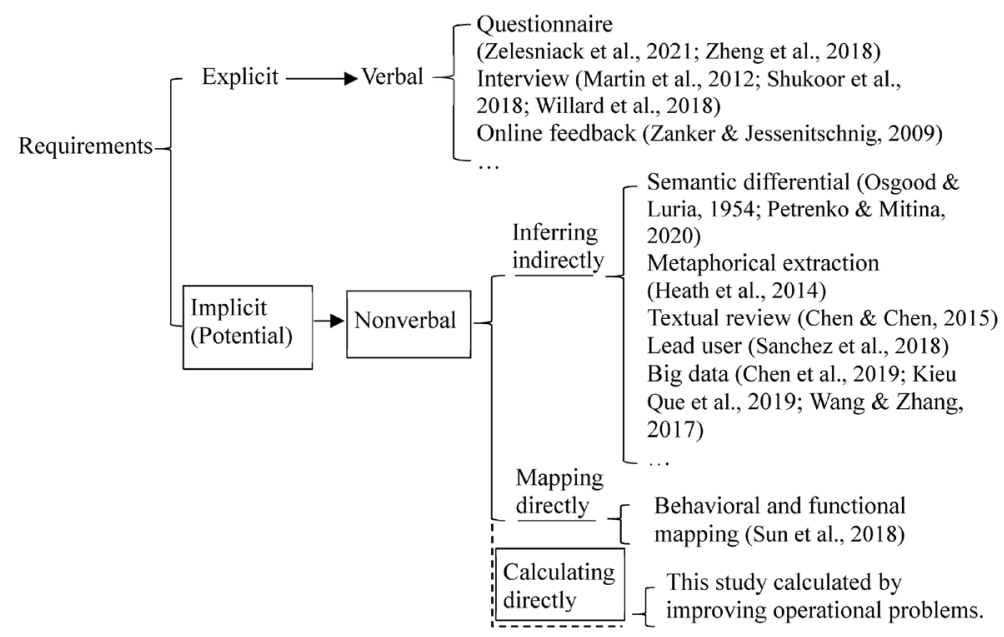

\section{METHODS}

The procedure (Figure 2) was to map the input to the output, where the input of the mining process is user operation information and the output is the potential requirement. Equipment, such as a camera, were used to collect operation information, following which the operation chain was constructed mathematically, problems during operation were modeled, and lastly, the mapping specifications 
from input to output were built. Here, a single task operation, which meant that a single goal was to be achieved within a specified time interval, was illustrated to explain the mining process because single tasks are one of the most common daily activities. This process included three key steps:

Figure 2. The process of user requirements mining based on operation optimization

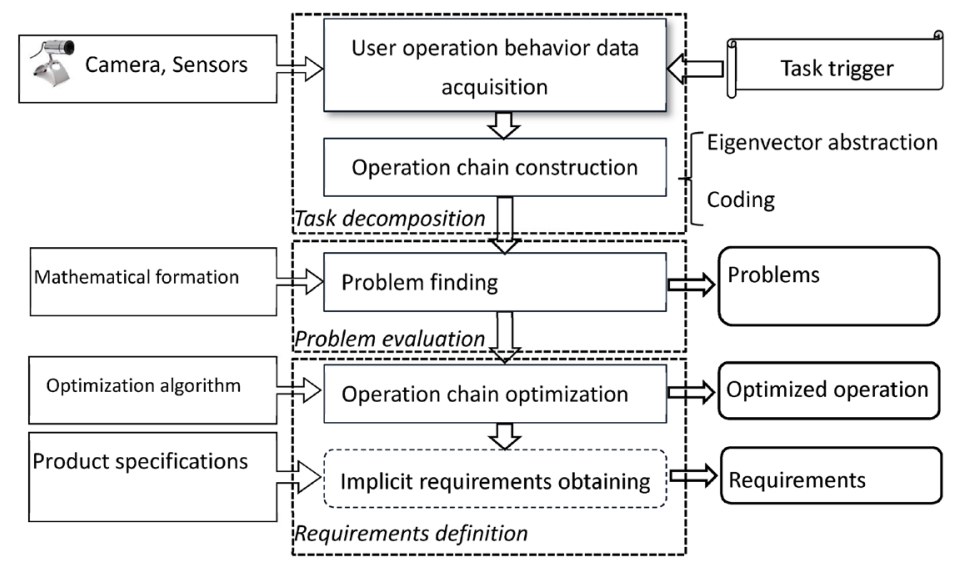

\section{Decomposing the Task and Capturing Users' Emotional and Behavioral Information}

The task was broken down into several suboperations and an operation chain including all these suboperations in sequence, in which each suboperation was an independent point in the chain. The task operation was camera-recorded for review to identify the problems.

\section{Expressing Operational Problems Mathematically}

Operational problems were quantitatively defined by the degree of physical difficulty, emotional state, and suboperation duration of each point in the operation chain.

\section{Defining Users' Potential Requirements}

To obtain the extreme value of the problems of each point defined in the second step, solutions that could be used to solve or minimize the emotional and behavioral problems in the operation chain were enumerated, and the operation chain was optimized. These solutions suggested users' real requirements and provided a more convenient and comfortable operation pattern.

\section{Decomposing the Task and Capturing Users' Emotional and Behavioral Information}

A user usually needs to complete a single task through several enforceable suboperations. In each suboperation, the user took an action and evaluated whether the subgoal was achieved, and then decided how and when to move forward to the next suboperation (Miller et al., 1960). In the laboratory, users' emotions and behaviors were recorded by cameras and sensors for evaluation (Figure 3) because the facial expression and eye movements (Lim et al., 2020) showed users' emotional state and body stress, while body movement indicated users' behavioral difficulty degrees. For example, the longer the duration of a suboperation, the more difficult it was to suboperate. Each suboperation was considered a point, and all the points of the task formed an entire operation chain. Information was captured for each point to calculate the degree of difficulty of the entire chain. Here, eye movement was labeled as 
,"and eye movements chain was like "

..," facial emotion state was labeled as “-,", and the facial emotional status chain was like "-_--_-_..;"while a single joint movement was labeled as " $i$." All captured joints movements are presented in Figure 3 since many joints moved together in suboperations. Each recorded performance was expressed mathematically using a representative index, which is explained in the following section.

Figure 3. Information acquisition and operation chain formation

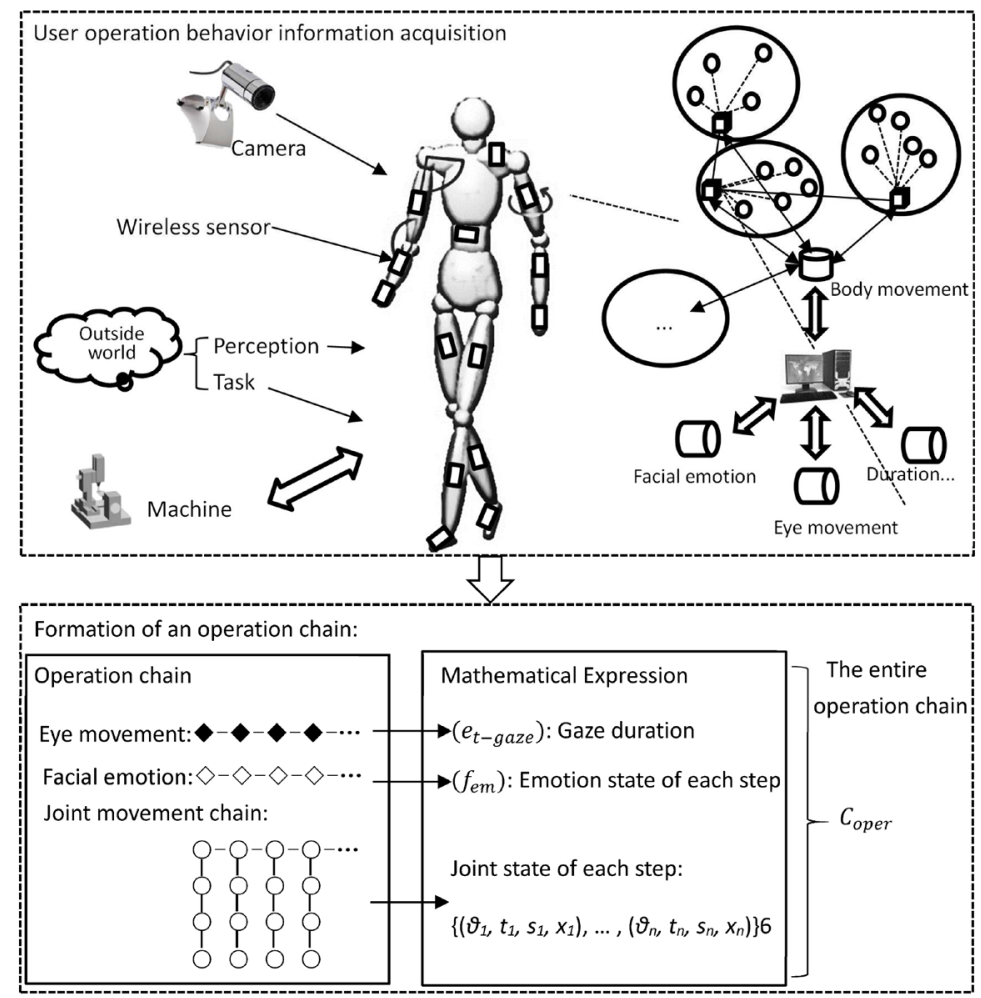

\section{Expressing Operational Problems Mathematically}

Figure 3 shows how users' behavioral and emotional data were captured by a camera, eye tracker, wireless force sensor, and displacement sensor while the user performed a task or interacted with a specific machine or product. During the task, users were encouraged to express their thoughts and feelings. For every suboperation in a task, the recorded data were normalized and stored in a central database. The data included two parts: emotional and physical information (Figure 4).

\section{Emotional Information}

Users' feelings, revealing whether the user was willing to perform the operation, could be expressed by eye movements and facial expressions. Thus, the gaze duration $\left(e_{t-g a z e}\right)$ was a key factor in eye movements because the longer a user gazed at an object, the more effort was needed in a suboperation. The facial expression $(f)$ was used to determine whether the user was feeling positive or negative. The emotions were roughly classified into eight categories: interested / excitement, happy / like, surprised, sad / painful, fearful, shy / humiliated, disgusted, and angry, which were represented by 


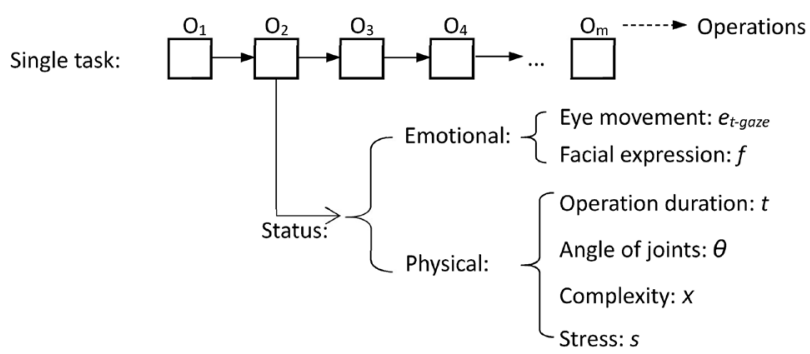

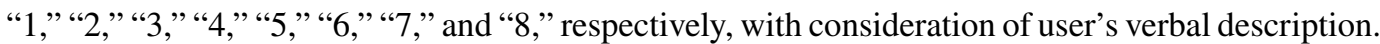
The bigger the number, the more negative the emotions were; for example, "surprised" valued " 3 " and "angry" valued "8." Therefore, the emotional state of the entire operation chain $E_{\text {emotion }}$ can be expressed as

$$
E_{\text {emotion }}=\left\{\left\{\left(e_{t-\text { gaze }}\right)_{t 0}, f_{t 0}\right\}, \ldots,\left\{\left(e_{t-g a z e}\right)_{t m}, f_{t m}\right\}\right\}_{(1)}
$$

where $e_{t \text {-gaze }}$ is the gaze duration in each suboperation, $m$ is the value of emotional status, $t$ indicates the duration of a suboperation, and $f$ is the facial state. Larger emotion values indicated more negative feelings than smaller ones. The standard value $e_{s}$, which means the maximum acceptable feelings for operating a task, is the mean value of $\left(e_{t-\text { gaze }}\right)_{t m}$ plus the neutral facial emotion value " 4 ":

$e_{s}=\frac{\left(e_{t-g a z e}\right)_{t m}}{m}+4$

\section{Physical Information}

As shown in Figure 4, physical behavioral difficulty is related to a motion's range, duration, complexity, and load, which are expressed by the joint flexion angle $(\theta)$, duration of suboperation $t$, muscle stress $s$, and complexity $x$, where muscle stress $s$ indicates the load carried by a muscle, and complexity $x$ is the number of body parts participating in the suboperation. For example, $x$ equals " 1 " when only one body part is involved such as a hand, and $x$ should be " 2 " when two body parts are involved, such as two hands, one hand, or one foot.

Therefore, the physical load state of the entire task chain, $B_{c}$, can be defined as

$$
B_{c}=\left\{\left[\begin{array}{c}
\left(\theta_{1}, t_{1}, s_{1}, x_{1}\right)_{t 0} \\
\left(\theta_{2}, t_{2}, s_{2}, x_{2}\right)_{t 0} \\
\ldots \\
\left(\theta_{n}, t_{n}, s_{n}, x_{n}\right)_{t 0}
\end{array}\right]\left[\begin{array}{c}
\left(\theta_{1}, t_{1}, s_{1}, x_{1}\right)_{t 1} \\
\left(\theta_{2}, t_{2}, s_{2}, x_{2}\right)_{t 1} \\
\ldots \\
\left(\theta_{n}, t_{n}, s_{n}, x_{n}\right)_{t 1}
\end{array}\right]\left[\begin{array}{c}
\left(\theta_{1}, t_{1}, s_{1}, x_{1}\right)_{t m} \\
\left(\theta_{2}, t_{2}, s_{2}, x_{2}\right)_{t m} \\
\ldots \\
\left(\theta_{n}, t_{n}, s_{n}, x_{n}\right)_{t m}
\end{array}\right]\right\}
$$


where $m$ is the number of suboperations, $n$ is the number of motion joints, and at moment $t_{0}$, the movement of one joint is $\left(\theta_{1}, t_{1}, s_{1}, x_{1}\right)_{t 0}$, and the movements of all motion joints at $t_{0}$ are the sums of the $t_{0}$ column.

\section{Defining Users' Potential Requirements}

Most users cannot express their potential requirements verbally (Leonard, 2013), while operational difficulties usually imply that improved operation patterns or product forms can help relieve users' emotional or physical burden. Therefore, defining users' potential requirements raised the need to find an optimal operation pattern/product to reduce users' physical and emotional stress, which could be transformed into a mathematical optimization according to Eq.1 and Eq.3.

Figure 5. Optimization of an operation chain by improving physical and emotional experiences

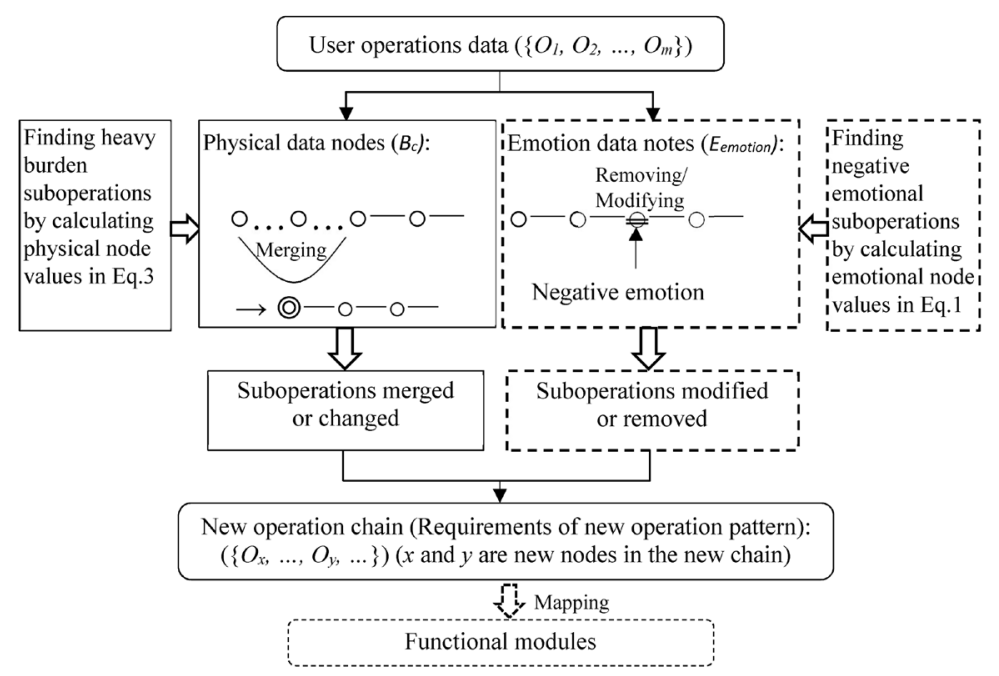

As shown in Figure 5, users' operation data were recorded and stored in a database and processed using the Python programming tool.

For the variable of emotional load, the goal was to improve negative emotions or replace negative points with positive ones, if possible. According to Eq.1, users' feelings were measured by their facial expressions and eye movements. First, the element values in $E_{\text {emotion }}$ were calculated; then, for equal or greater element values than a standard $e_{s}$ (Eq.2), the corresponding suboperations were marked for removal or modification, where a search engine was used to enumerate alternative modules with positive emotions from an existing database. For example, a case in the database suggested that some scents could generate positive feelings, so smell modules could be integrated into the operation chain as a substitute suboperation. These candidate modules formed multiple alternative operational chains.

For the physical load, the optimization goal was to minimize the value of $B_{c}$ in Eq.3. The summary values of each column indicate the physical loads for each suboperation/point. The first step was to manually check whether a set of adjacent points could be merged to reduce the point values. If "Yes," then alternative function modules could be selected from a database to substitute current function modules; and if "No," then we skipped to check the next set. This process was repeated until no adjacent points were merged. The second step was to adjust for the high- value points; for example, 
lowering the height of a worktable could consequently reduce the arm's stress. Consequently, new operational chains with reduced total point values are generated.

Therefore, a collection of alternative operational chains with reduced emotional or physical loads was obtained, where each new point in the generated chain representing a new operation was needed. Each operation could be mapped to several individual functional modules, for example, a rotating operation matched a knob or turntable, and the entire chain of functional modules representing the improved structures or operation patterns was generated. These structural schemes and operational patterns were then examined through practical manufacturing and ergonomic and economic specifications before moving into the detailed design stage. Using the mathematical optimization process, the users' potential requirements were discovered to improve user experiences.

\section{CASE STUDY}

\section{Experiment Design for User Data Collection}

One of the common daily menial activities for people is to hang garments after arriving home. We observed that coat hangers are usually installed at the entrances of most Chinese homes for family members to keep their outerwear, caps, or gowns. However, in reality, most people preferred to throw their outerwear somewhere nearby instead of hanging them using both hands, which indicated that throwing might relieve physical and emotional load, although it resulted in the room looking untidy and possibly increased levels of irritation in family members. The conflict between designers' expectations and users' behavior in this case implies that there are some potential requirements of the users.

It was assumed that there were some operational problems in hanging the clothes. An experiment was performed to measure users' emotional and physical loads for this activity. Twenty college students (10 males and 10 females, aged 18-24 years) were invited to participate in the experiment, as hanging clothes was an everyday task for all people, irrespective of their career and age. All participants signed a consent form prior to the experiment. These participants came from different parts of the country, had at least three years of experience of living alone, and were physically and psychologically healthy.

Figure 6. Experimental process of hanging clothes when users arrived home

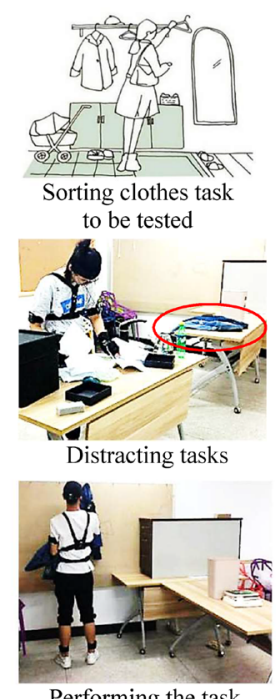

Performing the task

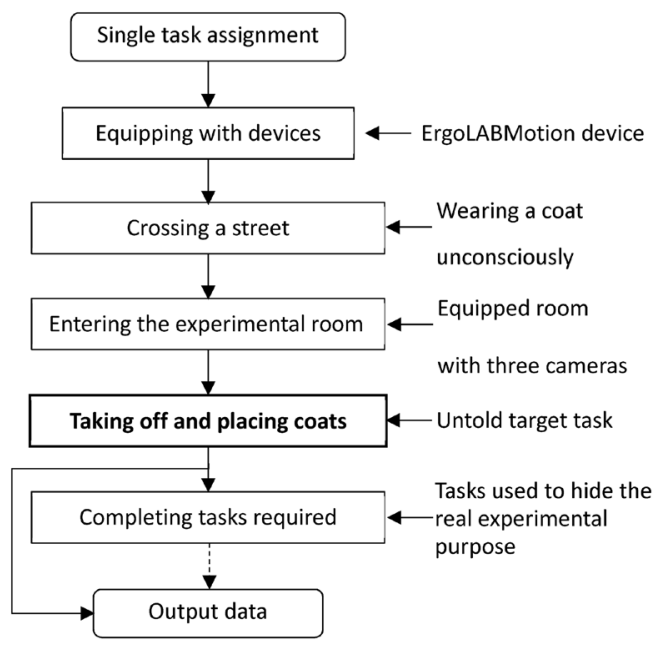


As shown in Figure 6, the participants were not informed of the actual purpose of the experiment. They were equipped with a wireless movement capture device (ErgoLAB Captiv Motion, Kinfar Limited Co.) at a resting place; then they put their coats on and entered a messy work studio. In the work studio, three cameras were set up at three different corners. To conceal the actual purpose of the experiment, the participants were assigned the task of cleaning the room. Their tasks included arranging the desks neatly, throwing all used water bottles into the trash can, and collecting scattered stationery into a container. Before they performed these tasks, they needed to take off their outerwear, and that was when the process of hanging clothes was captured by devices.

The main features of participant behavior were recorded for each suboperation without counting the muscle stress $s$ and the angle of the joints (in Eq. 3), because the activity was light physical labor. The gaze duration of a suboperation $\left(e_{t-g a z e}\right)$, main facial expression $\left(f_{e m}\right)$, suboperation duration $(t)$, maximum joint flexion angle of limbs $(\theta)$, and operation complexity $(x)$ were included.

\section{RESULTS AND VALIDATION}

The validation process included building the original operation chain, evaluating the problematic point, and optimizing the operation pattern by improving emotional and physical experiences.

First, according to the recorded data, the entire task of hanging clothes included five main suboperations: $\mathrm{O}_{1}, \mathrm{O}_{2}, \mathrm{O}_{3}, \mathrm{O}_{4}$, and $\mathrm{O}_{5}$. Table 1 lists the mean values for all the participants. The first column represents the names of each point in the operation chain, and each data column shows the captured operating status.

Table 1. Means and standard deviations of operation data for the clothes hanging task

\begin{tabular}{|c|c|c|c|c|c|c|}
\hline Suboperation & Description & $e_{t \text {-gaze }}(\mathbf{s})$ & $f_{e m}$ & $t(\mathbf{s})$ & $\Theta\left({ }^{\circ}\right)$ & $X$ \\
\hline$O_{1}$ & Walking and checking the task & $1.8(0.294)$ & $1(0)$ & $48.3(8.838)$ & $0(0)$ & $0(0)$ \\
\hline $\mathrm{O}_{2}$ & Taking off coats & $1.1(0.319)$ & $1(0)$ & $10.5(2.259)$ & $120.4(15.171)$ & $2(0)$ \\
\hline $\mathrm{O}_{3}$ & Looking around to put down coats & $0.4(0.097)$ & $2(0.816)$ & $1.4(0.275)$ & $0(0)$ & $1(0)$ \\
\hline $\mathrm{O}_{4}^{\prime}$ & Dropping it & $0.3(0.122)$ & $1(0)$ & 1.1(0.191) & $12.5(2.402)$ & $\mathbf{1}(\mathbf{0})$ \\
\hline$O_{4} "(1)$ & Moving to hangers & $0.3(0.119)$ & $2(0.756)$ & $7.6(1.397)$ & $0(0)$ & $1(0)$ \\
\hline $\mathrm{O}_{4}^{\prime \prime}(2)$ & Hanging coats & $2.6(0.469)$ & $2.8(0.463)$ & $13.3(3.147)$ & $171.8(23.787)$ & $2(0)$ \\
\hline $\mathrm{O}_{4}^{\prime \prime}(3)$ & Moving back to the assigned tasks & $0.4(0.151)$ & $2(0.535)$ & $9.3(1.799)$ & $0(0)$ & $0(0)$ \\
\hline $\mathrm{O}_{5}$ & Starting assigned tasks & \multicolumn{5}{|c|}{ (Moving to other tasks) } \\
\hline
\end{tabular}

Second, participants displayed positive "interest" or "excitement" when they came into the experimental work studio from Table 1 . All patients underwent operations from $O_{1}$ to $O_{3}$. While some chose $O_{4}$ ' and some chose $O_{4}$ " during the $O_{4}$, including $O_{4}$ " (1), $O_{4}$ ")(2), and $O_{4}{ }^{\prime}$ "(3). Each column shows significant differences when comparing $O_{4}$ ' and $O_{4}$ " in Table 1.

For gaze time, $e_{t \text {-gaze }}\left(O_{4}{ }^{\prime}\right)=e_{t \text {-gaze }}\left(O_{4}{ }^{\prime}(1)\right)+e_{t \text {-gaze }}\left(O_{4}{ }^{\prime}(2)\right)+e_{t \text {-gaze }}\left(O_{4}{ }^{\prime}(3)\right)$ $=0.3+2.6+0.4=3.3 \mathrm{~s}$,

while $e_{t \text {-gaze }}\left(O_{4}^{\prime}\right)=0.3 \mathrm{~s}(\mathrm{p}<0.001$, effect size Cohen's $\mathrm{d}=8.9746272)$.

Thus, $e_{\text {t-gaze }}\left(O_{4}\right.$ ”) outweighed $e_{t \text {-gaze }}\left(O_{4}^{\prime}\right)$, which implies a heavier mental load for $O_{4}$ ”" (hanging) than $O_{4}^{\prime}$ (throwing). 
In terms of facial expression $f_{e m}$, three participants showed "dislike" $\left(f_{e m}=7\right)$ and others remained "happy" $\left(f_{e m}=2\right)$; the mean value of $f_{e m}$ was 2.8 .

For physical variances, such as duration $(t)$, joint flexion angle of limbs $(\Theta)$, and operation complexity $(x)$, all proved that the burden of $O_{4}$ " was much higher than that of $O_{4}$ '. Here, the complexity $x$, the difficulty degree of each suboperation, was quantitatively calculated, and the original data were standardized to interval $[0,1]$ because the values of different variances had different measurements.

Finally, the total operational load of each task chain was obtained by adding the results of Eq. 1 and Eq.3, which show that the load of $\mathrm{O}_{4}$ "' (hanging) was significantly higher than $\mathrm{O}_{4}$ ' (throwing). This explains why many people preferred throwing clothes rather than hanging or folding them. The results also suggested that users needed improved products so that they could complete a task such as simply hanging clothes, with positive emotions and low stress. Therefore, users' potential requirement in this case was an improved design that provided an operation chain of: $\mathrm{O}_{1}, \mathrm{O}_{2}, \mathrm{O}_{3}$ and $O_{4}$, which meant that people needed a product that "allows them to drop / throw clothes by hand, sorts them automatically and neatly, and even helps releasing stress." These keywords revealed new requirement clues that could be used to design a new product.

\section{DISCUSSIONS AND CONCLUSIONS}

The results of the case showed that users' potential requirements for an operation pattern or a product could be calculated effectively from users' operational behaviors when the criterion was set on improving emotional experiences and relieving physical burden. This was also confirmed by the human-centered design theory (Norman, 2013) and human's innate pursuit for a happy and comfortable life.

In conclusion, this study differs from existing indirect extrapolating approaches (Lai et al., 2019; Liao et al., 2021; Shin et al., 2020) and contributes to providing a method for mining users' potential requirements directly from users' nonverbal behaviors, where users' demands for improving existing operation patterns or products were defined as requirements of emotion enhancement and physical burden relief during task performance. Taking a single task in daily life as an example, first, an operation was decomposed into several suboperations; second, the operations were modeled mathematically according to the evaluation of emotion and physical burden, and the users' potential requirements were obtained by optimizing the operational indices. This method could be effective in concept innovation or product design optimization for specific operation patterns or products compared with existing indirect inferring approaches, which are useful in predicting product trends. From this study, the following conclusions can be drawn:

(i) Users' operational behaviors and problems reflect their potential requirements.

(ii) Users' potential requirements can be mined while optimizing the task operation chain mathematically by improving the emotional and physical experiences.

(iii) The case in daily life proposed in this study showed the practicability of the potential requirements mining method, and it can be generalized to similar single tasks.

We believe that the method would be helpful for designers to discover users' potential requirements that users cannot consciously speak about specific products, and, as a result, make everyday life easier. However, this study has some limitations. First, there is a difference between actual daily activities and the experimental setting, such as the duration of each operation, which may have been affected because the experiment was performed in a work studio. However, the study findings present convincing evidence in discovering users' potential requirements by analyzing their operational behavior, as there were no differences between the two circumstances in the whole process, including the order of operations, feelings for actions, and the load comparison. In future studies, practical conditions 
need to be considered to evaluate the exact differences and refine the operation model. Second, it is not convenient for users to be equipped with measuring instruments in the real world if the task is complicated or dangerous. Remote sensors or simple wearables such as bracelets for measurement are required in the future. Third, the product scheme generation process requires further discussion in future research. In addition, users sometimes perform two or more tasks simultaneously, instead of a single task as discussed in this study. Experiments for multitask situations need to be discussed in future studies.

\section{ACKNOWLEDGMENT}

This research was supported by the National Natural Science Foundation of China (Grant No. 51875399), the Natural Science Foundation of Tianjin City (Grant No. 19JCYBJC19500), and the $1^{\text {st }}$ Batch of 2020 MOE of PRC Industry-University Collaborative Education Program (Program No. 202001SX03, Kingfar-CES "Human Factors and Ergonomics" Program). We would like to extend special acknowledgement to Editage (www.editage.cn) for English language editing. 


\section{REFERENCES}

Castellano-Tejedor, C., Moreno, J., Knittle, K., Nurmi, J., Ginchev, T., Parramon, G., Ciudin, A., Ramos-Quiroga, J. A., \& Lusilla-Palacios, P. (2020). Assessing the user experience and usability of the PRECIOUS system: A randomized controlled trial in obese patients. Informatics for Health \& Social Care, 45(4), 1-18. doi:10.1080/ 17538157.2020.1776292 PMID:32713290

Chen, G., \& Chen, L. (2015, August). Augmenting service recommender systems by incorporating contextual opinions from user reviews. User Modeling and User-Adapted Interaction, 25(3), 295-329. doi:10.1007/ s11257-015-9157-3

Chen, R., Wang, Q., \& Xu, W. (2019, November-December). Mining user requirements to facilitate mobile app quality upgrades with big data. Electronic Commerce Research and Applications, 38. Advance online publication. doi:10.1016/j.elerap.2019.100889

Cheng, Y.-M., \& Leu, S.-S. (2011, June). Integrating data mining with KJ method to classify bridge construction defects. Expert Systems with Applications, 38(6), 7143-7150. doi:10.1016/j.eswa.2010.12.047

Glass, A. S., Bagga, H. S., Tasian, G. E., McGeady, J. B., McCulloch, C. E., Blaschko, S. D., McAninch, J. W., $\&$ Breyer, B. N. (2013, August). No small slam: Increasing incidents of genitourinary injury from toilets and toilet seats. BJU International, 112(3), 398-403. doi:10.1111/bju.12173 PMID:23773285

Guo, Q., Xue, C., Yu, M., \& Shen, Z. (2019, March). A New User Implicit Requirements Process Method Oriented to Product Design. Journal of Computing and Information Science in Engineering, 19(1), 011010. Advance online publication. doi:10.1115/1.4041418

Hansen, J.-T., \& Rosen, D. W. (2019, September). A Product Family Design Method for Configuration and Spatial Layout Requirements. Journal of Computing and Information Science in Engineering, 19(3), 031008. Advance online publication. doi:10.1115/1.4042300

Heath, D., Norton, D., \& Ventura, D. (2014, April). Conveying Semantics through Visual Metaphor. ACM Transactions on Intelligent Systems and Technology, 5(2), 31. Advance online publication. doi:10.1145/2589483

Kakar, A. S. (2016, January-March). A User-Centric Typology of Information System Requirements. Journal of Organizational and End User Computing, 28(1), 32-55. doi:10.4018/JOEUC.2016010103

Kieu Que, A., Nagai, Y., \& Nguyen Le, M. (2019). Extracting user requirements from online reviews for product design: A supportive framework for designers. Journal of Intelligent \& Fuzzy Systems, 37(6), 7441-7451. doi:10.3233/JIFS-179352

Klettner, S. (2020, May). Affective Communication of Map Symbols: A Semantic Differential Analysis. ISPRS International Journal of Geo-Information, 9(5), 289. Advance online publication. doi:10.3390/ijgi9050289

Lai, X., Zhang, Q., Chen, Q., Huang, Y., Mao, N., \& Liu, J. (2019, September 17). The analytics of productdesign requirements using dynamic internet data: Application to Chinese smartphone market. International Journal of Production Research, 57(18), 5660-5684. doi:10.1080/00207543.2018.1541200

Leonard, M. (2013). Subliminal: how your unconscious mind rules your behavior. Vintage Books.

Liao, X., Ye, G., Yu, J., \& Xi, Y. (2021, May). Identifying lead users in online user innovation communities based on supernetwork. Annals of Operations Research, 300(2), 515-543. doi:10.1007/s10479-021-03953-0

Lim, J. Z., Mountstephens, J., \& Teo, J. (2020, April). Emotion Recognition Using Eye-Tracking: Taxonomy, Review and Current Challenges. Sensors (Basel), 20(8), 2384. Advance online publication. doi:10.3390/ s20082384 PMID:32331327

Manasrah, A. M., Aldomi, A., \& Gupta, B. B. (2019, January). An optimized service broker routing policy based on differential evolution algorithm in fog/cloud environment. Cluster Computing-the Journal of Networks Software Tools and Applications, 22(S1), 1639-1653. doi:10.1007/s10586-017-1559-z

Martin, J. L., Clark, D. J., Morgan, S. P., Crowe, J. A., \& Murphy, E. (2012, January). A user-centred approach to requirements elicitation in medical device development: A case study from an industry perspective. Applied Ergonomics, 43(1), 184-190. doi:10.1016/j.apergo.2011.05.002 PMID:21636072 
Miller, G. A., Gallanter, E., \& Pribram, K. H. (1960). Plans and the Structure of Behavior. Holt, Rinehart \& Winston. doi:10.1037/10039-000

Norman, D. A. (2013). Design of everyday things. MIT Press.

Osgood, C. E., \& Luria, Z. (1954). 1954-Oct). A blind analysis of a case of multiple personality using the semantic differential. Journal of abnormal psychology, 49(1, Part 1), 579-591. doi:10.1037/h0054362 PMID:13201293

Pauligk, S., Kotz, S. A., \& Kanske, P. (2019, October 8). Differential Impact of Emotion on Semantic Processing of Abstract and Concrete Words: ERP and fMRI Evidence. Scientific Reports, 9(1), 14439. Advance online publication. doi:10.1038/s41598-019-50755-3 PMID:31594966

Petrenko, V., \& Mitina, O. (2020, July). The Fairytale Semantic Differential Technique: A Cross-Cultural Application. Behavioral Sciences (Basel, Switzerland), 10(7), 112. Advance online publication. doi:10.3390/ bs10070112 PMID:32640714

Sanchez, V. V. S., Giacalone, D., \& Goduscheit, R. C. (2018, March). Digital anthropology as method for lead user identification from unstructured big data. Creativity and Innovation Management, 27(1), 32-41. doi:10.1111/ caim. 12228

Sen, G., \& Sener, B. (2020). Design for Luxury Front-Seat Passenger Infotainment Systems with Experience Prototyping through VR. International Journal of Human-Computer Interaction, 36(18), 1714-1733. Advance online publication. doi:10.1080/10447318.2020.1785150

Shin, G. W., Park, S., Kim, Y. M., Lee, Y., \& Yun, M. H. (2020, July). Comparing Semantic Differential Methods in Affective Engineering Processes: A Case Study on Vehicle Instrument Panels. Applied Sciences-Basel, 10(14), 4751. Advance online publication. doi:10.3390/app10144751

Shukoor, N. S. M., Tamrin, S. B. M., Guan, N. Y., \& Nata, D. H. M. S. (2018). Development of new hard hat dimensions using user-centered design approach among oil palm harvesters. Work (Reading, Mass.), 60(1), 129-134. doi:10.3233/WOR-182741 PMID:29843301

Silva-Rodriguez, V., Edith Nava-Munoz, S., Castro, L. A., Martinez-Perez, F. E., Perez-Gonzalez, H. G., \& TorresReyes, F. (2020, October). Classifying design-level requirements using machine learning for a recommender of interaction design patterns. IET Software, 14(5), 544-552. doi:10.1049/iet-sen.2019.0291

Sun, X., Houssin, R., Renaud, J., \& Gardoni, M. (2018). Towards a human factors and ergonomics integration framework in the early product design phase: Function-Task-Behaviour. International Journal of Production Research, 56(14), 4941-4953. doi:10.1080/00207543.2018.1437287

Turan, H., Saricaoglu, H., Turan, A., \& Tunali, S. (2011, November-December). Polyurethane toilet seat contact dermatitis. Pediatric Dermatology, 28(6), 731-732. doi:10.1111/j.1525-1470.2011.01482.x PMID:21575050

van Haasteren, A., Vayena, E., \& Powell, J. (2020, July). The Mobile Health App Trustworthiness Checklist: Usability Assessment. JMIR mHealth and uHealth, 8(7), e16844-e16844. doi:10.2196/16844 PMID:32706733

Wang, H., \& Zhang, H. (2017). User Requirements Based Service Identification for Big Data. 10.1109/ ICWS.2017.11

Willard, S., Cremers, G., Man, Y. P., van Rossum, E., Spreeuwenberg, M., \& de Witte, L. (2018, April 7). Development and testing of an online community care platform for frail older adults in the Netherlands: A user-centred design. BMC Geriatrics, 18(1), 87. Advance online publication. doi:10.1186/s12877-018-0774-7 PMID:29625562

Zanker, M., \& Jessenitschnig, M. (2009, February). Case-studies on exploiting explicit customer requirements in recommender systems. User Modeling and User-Adapted Interaction, 19(1-2), 133-166. doi:10.1007/s11257008-9048-y

Zelesniack, E., Oubaid, V., \& Harendza, S. (2021, June 5). Final-year medical students' competence profiles according to the modified requirement tracking questionnaire. BMC Medical Education, 21(1), 319. Advance online publication. doi:10.1186/s12909-021-02728-2 PMID:34088296

Zheng, H., Rosal, M. C., Li, W., Borg, A., Yang, W., Ayers, D. C., \& Franklin, P. D. (2018, April). A Web-Based Treatment Decision Support Tool for Patients With Advanced Knee Arthritis: Evaluation of User Interface and Content Design. JMIR Human Factors, 5(2), e17-e17. doi:10.2196/humanfactors.8568 PMID:29712620 
Yanqun Huang has a Ph.D and is an Associate Professor in the Key Laboratory of Mechanism Theory and Equipment Design of Ministry of Education at Tianjin University, Tianjin, China and Tianjin Ren'ai College, Tianjin, China. Her research interests include Industrial Design, Design Psychology, User Research and Human-machine interaction.

Gaofeng Pan is a Ph.D candidate in the School of Mechanical Engineering at Tianjin University, Tianjin, China and Tianjin Tianduan Press Co. Ltd., Tianjin, China. He is a Senior Manager of Technology, and his research area is Product Design.

$\mathrm{Xu} \mathrm{Li}$ is a lecturer in the School of Mechanical Engineering at Tianjin Polytechnic University, China. His research area is User Research and Product Design.

Zhe Sun is a master student in the School of Management at Tianjin University, Tianjin, China. He is studying for the Master Degree in Industrial Engineering.

Shinichi Koyama has a Ph.D and is a Professor in the Faculty of Art and Design at University of Tsukuba, Japan. His research area is Design Psychology.

Yanqun Yang is an Associate Professor in the School of Civil Engineering at Fuzhou University, Fuzhou, China. His research area is Traffic design and safety. 